Retinoic acid and cAMP inhibit rat hepatocellular carcinoma cell proliferation and enhance cell differentiation

M. Ionta, M.C. Rosa, R.B. Almeida, V.M. Freitas, P. Rezende-Teixeira and G.M. Machado-Santelli

The Brazilian Journal of Medical and Biological Research is partially financed by

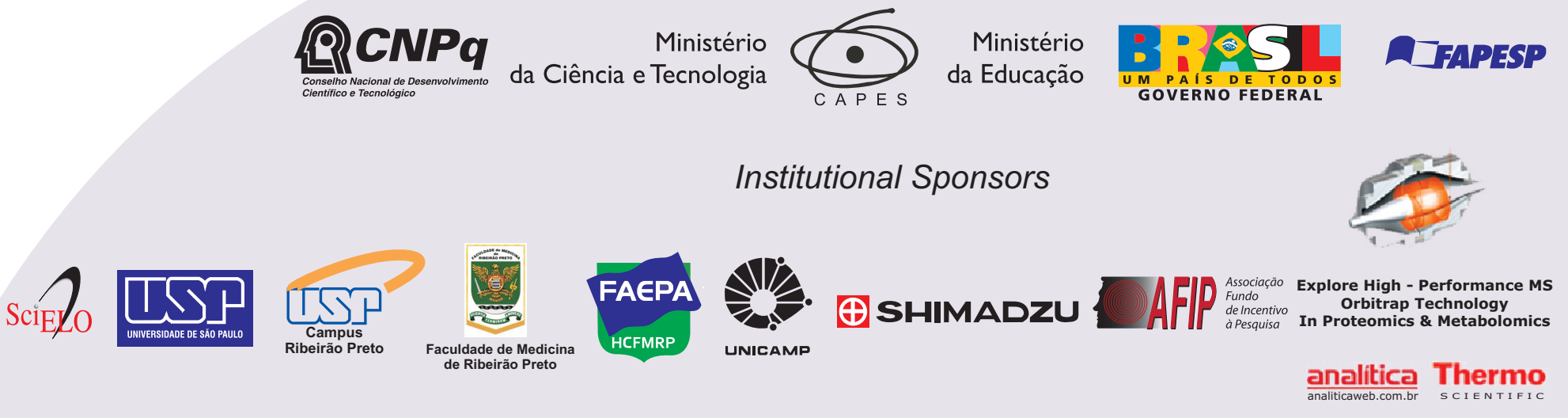




\title{
Retinoic acid and cAMP inhibit rat hepatocellular carcinoma cell proliferation and enhance cell differentiation
}

\author{
M. Ionta ${ }^{1,2}$, M.C. Rosa ${ }^{2}$, R.B. Almeida ${ }^{2}$, V.M. Freitas ${ }^{2}$, P. Rezende-Teixeira ${ }^{2}$ and \\ G.M. Machado-Santelli ${ }^{2}$ \\ ${ }^{1}$ Instituto de Ciências Biomédicas, Universidade Federal de Alfenas, Alfenas, MG, Brasil \\ 2Departamento de Biologia Celular e do Desenvolvimento, Instituto de Ciências Biomédicas, \\ Universidade de São Paulo, São Paulo, SP, Brasil
}

\begin{abstract}
Hepatocellular carcinoma (HCC) is the third highest cause of cancer death worldwide. In general, the disease is diagnosed at an advanced stage when potentially curative therapies are no longer feasible. For this reason, it is very important to develop new therapeutic approaches. Retinoic acid (RA) is a natural derivative of vitamin A that regulates important biological processes including cell proliferation and differentiation. In vitro studies have shown that RA is effective in inhibiting growth of HCC cells; however, responsiveness to treatment varies among different $\mathrm{HCC}$ cell lines. The objective of the present study was to determine if the combined use of RA $(0.1 \mu \mathrm{M})$ and cAMP $(1 \mathrm{mM})$, an important second messenger, improves the responsiveness of HCC cells to RA treatment. We evaluated the proliferative behavior of an HCC cell line (HTC) and the expression profile of genes related to cancer signaling pathway (ERK and GSK-3 $\beta$ ) and liver differentiation [E-cadherin, connexin 26 (Cx26), and connexin 32 (Cx32)]. RA and cAMP were effective in inhibiting the proliferation of HTC cells independently of combined use. However, when a mixture of RA and cAMP was used, the signals concerning the degree of cell differentiation were increased. As demonstrated by Western blot, the treatment increased E-cadherin, Cx26, Cx32 and Ser9-GSK-3 $\beta$ (inactive form) expression while the expression of Cx43, Tyr216-GSK-3 $\beta$ (active form) and phosphorylated ERK decreased. Furthermore, telomerase activity was inhibited along treatment. Taken together, the results showed that the combined use of RA and cAMP is more effective in inducing differentiation of HTC cells.
\end{abstract}

Key words: Cell adhesion; cAMP; Differentiation; Proliferation; Hepatocarcinoma; Retinoic acid

\section{Introduction}

Hepatocellular carcinoma (HCC) is a major healthcare problem worldwide representing the fifth leading cause of cancer mortality and the third most common cause of cancer-related deaths (1). The development of HCC is frequently associated with chronic viral infection (hepatitis $B$ virus or hepatitis $\mathrm{C}$ virus); however, frequent exposure to toxic agents (alcohol or aflatoxin) can contribute to the development of HCC (2).

In general, HCC is diagnosed in advanced stages when potentially curative therapies are not feasible. Only a minority of HCC are detected at an early enough stage for potentially curative therapies, such as surgical resection, liver transplantation or local ablation therapy. Thus, the development of alternative therapeutic approaches to
$\mathrm{HCC}$ remains one of the most challenging areas of cancer research.

Retinoic acid (RA) is an active metabolite of vitamin A that regulates important biological processes such as cell proliferation, cell differentiation and cell death (3). It has been reported that $\mathrm{RA}$ inhibits the proliferation of malignant tumor cells; however, its negative effect on cell growth is dose-dependent and cell-specific (4).

It is well known that RA not only induces growth inhibition of leukemia cells, but also stimulates their maturation. RA has been used as a chemotherapeutic agent in patients with acute promyelocytic leukemia (APL) since 1985 (5). Since then, the activity of RA on cell proliferation has been investigated in tumor cells derived from solid tumors, in-

Correspondence: G.M. Machado-Santelli, Departamento de Biologia Celular e do Desenvolvimento, ICB, USP, Av. Prof. Lineu Prestes, 1524, ICB I, Sala 306/307, 05508-000 São Paulo, SP, Brasil. Fax: +55-11-3091-7402. E-mail: glaucia.santelligmail.com

Received November 26, 2011. Accepted April 27, 2012. Available online May 25, 2012. Published August 3, 2012. 
cluding HCC (6-8).

Jung et al. (9) carried out an interesting study working with 4 different HCC cell lines (HepG2, SNU354, Hep3B, and SNU449). They verified that RA inhibited cell growth in only two cell lines (HepG2 and SNU354). Curiously, the proliferative behavior of Hep3B and SNU449 cells was stimulated by treatment with $\mathrm{RA}$. These results clearly show that HCC present distinct responsiveness to RA depending on their characteristics.

The combined use of two or more drugs can be advantageous in chemotherapy. Drug dosage would be lower, thus decreasing toxicity. Furthermore, a combination of drugs may improve treatment responsiveness through synergistic effects.

It was reported that RA induces the maturation of APL cells when used at concentrations close to physiological levels (1.0 to $10 \mathrm{nM})$ due to a sustained increase of the endogenous level of cAMP (10). cAMP is a second messenger that can act as a pro- or anti-mitotic agent depending of both cell type and the physiological state of the cell. The antiproliferative activity of CAMP on HCC cells has been demonstrated in some studies. Lee et al. (11) showed G1 synchronization of HepG2 cells due to down-regulation of cyclin A expression. In BEL-7402 cells, cAMP induced Akt inactivation with consequent inhibition of proliferation (12). Using a rat model of HCC, Kovac et al. (13) demonstrated that CAMP can inhibit cell mitogenesis.

The objective of the present study was to investigate the effects of the combined use of RA and cAMP on the proliferative behavior and phenotype of an HTC cell line derived from rat HCC. Our results showed that both RA and CAMP inhibit the proliferation of HTC cells. Moreover, the period of inhibition was greater in cells that received the mixture than in those treated with either RA or cAMP alone. Another advantage of the RA and cAMP combination is the degree of differentiation achieved. HTC cells treated with the drug mixture presented a more differentiated phenotype. Thus, the combined use of RA and cAMP extended growth inhibition and increased the degree of differentiation of HCC cells.

\section{Material and Methods}

\section{Cell culture conditions}

The rat HCC cell line (HTC) used in this study was acquired from the Cell Bank of Rio de Janeiro (14). The cultures were maintained in Dulbecco's modified Eagle's minimum essential medium (DMEM; Sigma, USA) supplemented with $5 \%$ fetal bovine serum (FBS; Cultilab, Brazil). Cells were grown at $37^{\circ} \mathrm{C}$ in a humidified incubator containing $5 \% \mathrm{CO}_{2}$ and subcultured every 2 or 3 days.

\section{Drugs and treatment schedule}

Stock solutions of $1 \mathrm{mM}$ RA(Sigma) and 100 mM cAMP
(Sigma) were prepared and diluted in DMEM (supplemented with $5 \%$ FBS) to a final concentration of $0.1 \mu \mathrm{M}$ RA and 1 $\mathrm{mM}$ cAMP. Drugs were used alone or in combination and were added on the 3rd day after plating. The treatment schedule was standardized using BRL3A, a non-tumoral cell line that presents great responsiveness to the drugs used (Figure 1). HTC cells were treated for 2 days (T0). After this period, the medium was replaced with drug-free medium and the cultures were tested for recovery at 24 (T24) and $48 \mathrm{~h}$ (T48).

\section{Bromodeoxyuridine (BrdU) incorporation}

Cells were cultured on coverslips in $35-\mathrm{mm}$ dishes at 5 $\times 10^{3}$ cells/plate. On the testing days, $100 \mu \mathrm{MBrdU}$ (Sigma)

\section{Control}
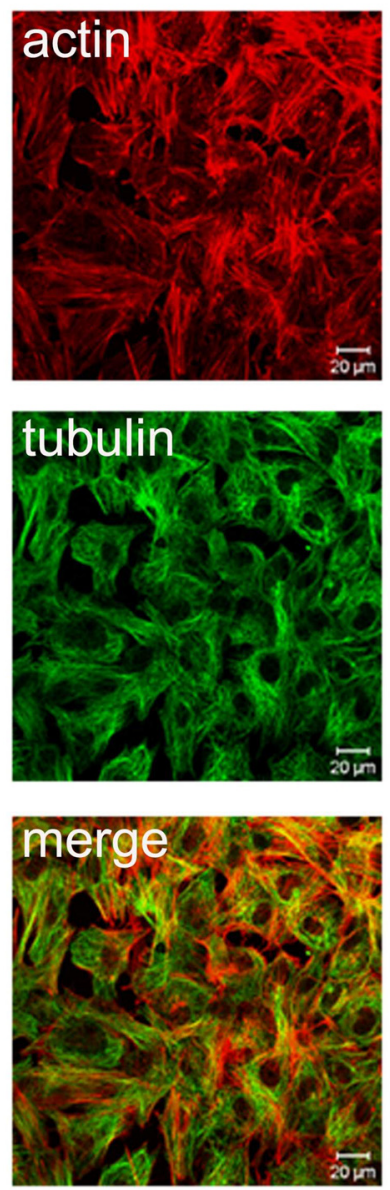

$$
\mathrm{RA}+\mathrm{cAMP}
$$
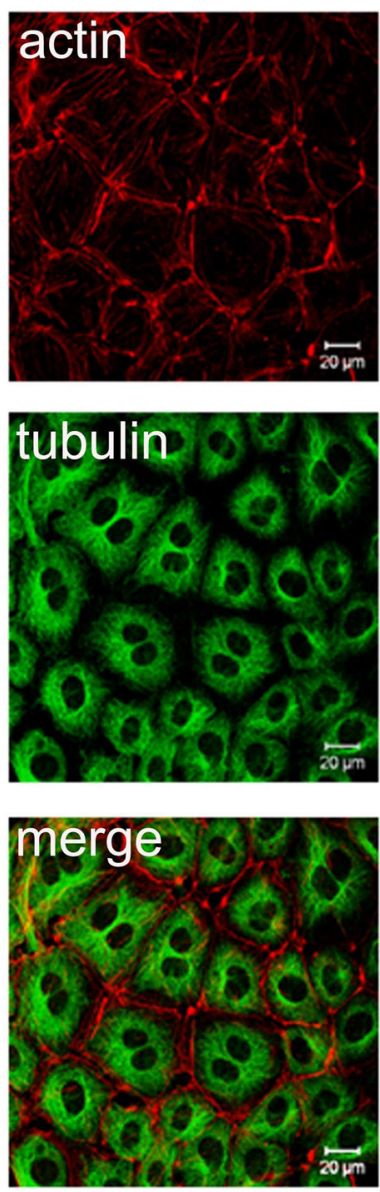

Figure 1. Laser scanning confocal microscopy images of BRL3A cells showing the actin filament distribution pattern at T48. The morphology of cells is demonstrated by F-actin staining (red) and anti-tubulin immunoreaction (green). Retinoic acid (RA) and cAMP induced cytoskeleton reorganization and a change in cell shape to polyhedric. Note the presence of binucleated cells in the images that indicate microtubule networks. 
was added to the medium for $1 \mathrm{~h}$. The cells were fixed in ethanol:acetic acid (3:1) for $30 \mathrm{~min}$ and the immunofluorescence assay was performed as described by Prado et al. (15). Mouse anti-BrdU antibody (Sigma) was incubated (1 h) in a wet chamber at room temperature. After washes in Dulbecco's phosphate-buffered saline (PBSA), the secondary antibody (anti-mouse IgG-FITC, Sigma) was incubated for $2 \mathrm{~h}$. The nuclei were stained with $10 \mu \mathrm{g} / \mathrm{mL}$ propidium iodide and the coverslips were mounted on histological slides with Vecta-Shield (Vector Laboratories, USA). The analyses were performed using fluorescence microscopy (Zeiss, Germany). One thousand cells were counted in each sample. Three independent experiments were performed and the data are reported as means \pm SD.

\section{Mitotic index}

Cells were cultured on coverslips in $35-\mathrm{mm}$ dishes at $5 \times 10^{3}$ cells/plate. On the indicated days cells were fixed in ethanol:acetic acid (3:1) for $30 \mathrm{~min}$ at room temperature and stained by the Feulgen reaction as described by Machado-Santelli et al. (16). Metaphase frequency was determined in one thousand cells counted in each sample. Data are reported as means \pm SD for three independent experiments.

\section{Cytoskeleton elements: microtubules and F-actin}

Cells were cultured on coverslips in 35-mm dishes at 5 $x 10^{3}$ cells/plate. Cells were fixed with $3.7 \%$ formaldehyde for $30 \mathrm{~min}$, and the cell membrane was permeabilized with $0.5 \%$ Triton X-100 for $10 \mathrm{~min}$. Cells were incubated overnight with mouse monoclonal primary antibodies (anti- $\alpha$ and anti- $\beta$ tubulin diluted in PBSA at 1:200 and 1:100, respectively) in a wet chamber at $4^{\circ} \mathrm{C}$. A secondary antibody (anti-mouse IgG-FITC, Sigma) was added for $2 \mathrm{~h}$ and the microfilaments were stained with TRITC-phalloidin for 20 min. Coverslips were mounted on histological slides with Vecta-Shield (Vector Laboratories). The analyses were performed using a confocal laser scanning microscope (LSM 510, Zeiss). The lasers used were argon (488 nm) and helium-neon (543 $\mathrm{nm}$ ) connected to an inverted fluorescence microscope (Axiovert 100M, Zeiss). Binucleated cells were quantified in fluorescent preparations by analyzing 1000 cells per slide. Data are reported as means \pm SD for three independent experiments.

\section{PCR-ELISA telomerase assay}

Quantitative determination of telomerase activity was performed according to the Telomeric Repeat Amplification Protocol using the TeloTAGGG Telomerase PCR ELISAPLUS kit (Roche Diagnostic GmbH, Germany). Briefly $2 \times 10^{5}$ cells were lysed using $2 \mathrm{~mL}$ lysis buffer and centrifuged at $4^{\circ} \mathrm{C}$ for $30 \mathrm{~min}$ at $13,000 \mathrm{rpm}$. The supernatants of different samples were divided into two aliquots before performing the assay: one was used as negative control after heat inactivation of telomerase at $80^{\circ} \mathrm{C}$ for $10 \mathrm{~min}$ and the other was used to evaluate the telomerase-mediated addition of the telomeric sequence. The products obtained were amplified by PCR. To exclude false-negative results due to Taq DNA-polymerase inhibitors eventually present in the lysates, a 216-bp long internal standard, present in the reaction mixture, was simultaneously amplified. The results are reported as relative telomerase activity (RTA), which was calculated according to the following formula: RTA = [(Abs sample - Abs RNAase-treated sample) / Abs internal standard of the sample] / [(Abs control template - Abs lysis buffer) / Abs internal standard of the control template] x 100 , where Abs = absorbance.

\section{Immunoblotting}

Cells were cultured in $75-\mathrm{cm}^{2}$ flasks, washed with cold PBSA and scraped into radioimmunoprecipitation assay buffer (150 mM NaCl, 1.0\% Nonidet P-40, 0.5\% deoxycholate, $0.1 \%$ SDS, and $50 \mathrm{mM}$ Tris, $\mathrm{pH} 8.0$ ) with both protease and phosphatase inhibitors (Sigma). Lysates were centrifuged at $10,000 \mathrm{~g}$ for $10 \mathrm{~min}$ at $4^{\circ} \mathrm{C}$. The supernatants were recovered, protein was quantified using a bicinchoninic acid kit (Pierce Biotechnology Inc., USA) and resuspended in Laemmli sample buffer containing $62.5 \mathrm{mM}$ Tris- $\mathrm{HCl}, \mathrm{pH} 6.8$, $2 \%$ SDS, $10 \%$ glycerol, $5 \%$ mercaptoethanol, and $0.001 \%$ bromophenol blue. A 30- $\mu$ g protein aliquot was separated by SDS-PAGE (10\%) and transferred (100 V, $250 \mathrm{~mA}$ for $2 \mathrm{~h}$ ) to a polyvinylidene fluoride membrane (Amersham Bioscience, USA). The blocking solution (Tris-buffered saline and $0.05 \%$ Tween $20=$ TTBS plus $5 \%$ skim milk) was added at $4{ }^{\circ} \mathrm{C}$ for $1 \mathrm{~h}$. The membrane was probed with different antibodies [anti-connexin 43 (Cx43) mouse monoclonal antibody, Sigma (1:500); anti-Cx32 mouse monoclonal antibody, Sigma (1:300); rabbit polyclonal anti-Cx26 antibody, Sigma (1:500); anti-vimentin, Chemicon (1:300, USA); anti-pERK (phosphorylated extracellular signal-regulated kinase), anti-ERK, anti-Ser9-pGSK-3 $\beta$ (phosphorylated glycogen synthase kinase 3 beta), anti-Tyr216-pGSK-3 $\beta$ and antiE-cadherin antibodies, Santa Cruz (1:500, USA)]. All blots were incubated with the antibodies overnight. After washing with TTBS, anti-mouse or anti-rabbit peroxidase-conjugated antibodies were added for $2 \mathrm{~h}$ at room temperature. The immunoreactive bands were visualized with the ECL Western blotting detection kit (Amersham Pharmacia) according to manufacturer instructions. Anti- $\alpha$-tubulin antibody was used as control to check loading conditions.

\section{Cell adhesion assay}

Adhesion assay was performed on 96-well roundbottomed plates. Wells were coated with $100 \mu \mathrm{L}$ laminin- 111 ( $1 \mu \mathrm{g} /$ well) overnight at $4{ }^{\circ} \mathrm{C}$. Wells were blocked with $3 \%$ BSA for 30 min at $37^{\circ} \mathrm{C}$ and rinsed in $0.1 \%$ BSA. The cells were maintained in a humidified incubator containing $5 \%$ $\mathrm{CO}_{2}$ at $37^{\circ} \mathrm{C}$ for $20 \mathrm{~min}$. After attachment, the cells were fixed/stained for $10 \mathrm{~min}$ with $0.2 \%(\mathrm{w} / \mathrm{v})$ crystal violet in $20 \%(\mathrm{v} / \mathrm{v})$ methanol. After three washes with $\mathrm{H}_{2} \mathrm{O}$, cells 
were dissolved in $10 \%$ (w/v) SDS, and the absorbance at $600 \mathrm{~nm}$ was measured. The experiment was performed in triplicate and data are reported as means \pm SD.

\section{Statistical analysis}

The quantitative data are reported as means \pm SD. ANOVA was performed and the experiments were carried out in triplicate. We also applied the Bonferroni multiple comparisons test. The Graphpad Prism software (GraphPad Software, Inc., USA) was used.

\section{Results}

\section{Standardization of the treatment schedule}

To determine the treatment schedule, we used the BRL3A cell line, a lineage established from rat normal liver. The assays performed with BRL3A cells showed that RA alone was able to induce cell differentiation; however, the cell population with a differentiated phenotype was more expressive when RA was associated with cAMP. We observed drastic changes in morphology of BRL3A cells as well as a significant increase in the frequency of binucleated cells, an important indicator of a differentiated phenotype in hepatic cells. Thus, we used the same treatment schedule for HTC cells, e.g., $48 \mathrm{~h}$ of treatment (RA and cAMP used in combination or not) followed by $24-$ and 48 -h recovery in fresh medium.

\section{Cell proliferation}

HTC cells treated with RAand/or cAMP for $48 \mathrm{~h}$ exhibited a decrease in cell proliferation, as demonstrated by BrdU incorporation and mitotic index (Figure 2). Immediately after the treatments (TO) the mitotic indexes were lower than control independently of the type of treatment $(3.7 \%$ for the control, $2.5 \%$ for RA, $1.84 \%$ for cAMP, and $1.78 \%$ for RA plus CAMP). After $24 \mathrm{~h}$ of recovery (T24), the mitotic index reached $5.6 \%$ in controls as opposed to $0.6,2.63$, and $2.16 \%$ for cells treated with RA, cAMP and RA plus cAMP, respectively. At T48, the cells seemed to recover their proliferation capacity, and the mitotic indexes of the treated cells were similar to control. The percentage of cells in the S-phase at T0 was higher in the control culture (33.76\%) compared to treated cultures $(24.1 \%$ in RA, $20 \%$ in cAMP, and $20 \%$ in RA plus cAMP). After a $24-\mathrm{h}$ recovery, BrdU indexes were similar for cAMP-treated and control cells while the S-phase indexes for RA or cAMP plus RA cells remained lower. After a 48-h recovery, the only difference was observed in the RA-treated group, with no differences among the other three groups.

\section{Cell differentiation}

Morphology and adhesion capacity. HTC is a pleomorphic cell line, which contains cells with different morphological features. This aspect is probably related to differences in cell-to-substrate adhesion levels, as indicated by the observation of flattened cells that adhere to the substrate and rounded cells that grow only partially adhered to the substrate (Figure $3 \mathrm{~A}$ and B). Figure 3 shows different optical sections obtained by confocal LSM of cytological preparations stained with TRITC-phalloidin at T48. In the treated cultures, we only observed cells growing tightly adhered to the substrate (Figure $3 \mathrm{C}-\mathrm{H}$ ). These cells exhibited actin stress fibers that were widely distributed in the cytoplasm (Figure 3C, E and G). The pattern of microfilament distribution in the treated cells was very similar to that observed in BRL3A cells, a non-tumoral liver cell line (data not shown). Another important aspect of treated cultures was the cellular arrangement. The cells treated with RA and RA plus cAMP (Figure 3 C,D and G,H) were arranged in a rope-like manner. Interestingly, cAMP treatment increased the frequency of binucleated cells (Figure 3E and F). At T48 (the time point of immunofluorescent confocal images), $0.3 \%$ of cells were binucleated in control cultures compared to $0.5,1.1$, and $1.13 \%$ in cultures treated with RA, cAMP and RA plus cAMP, respectively.
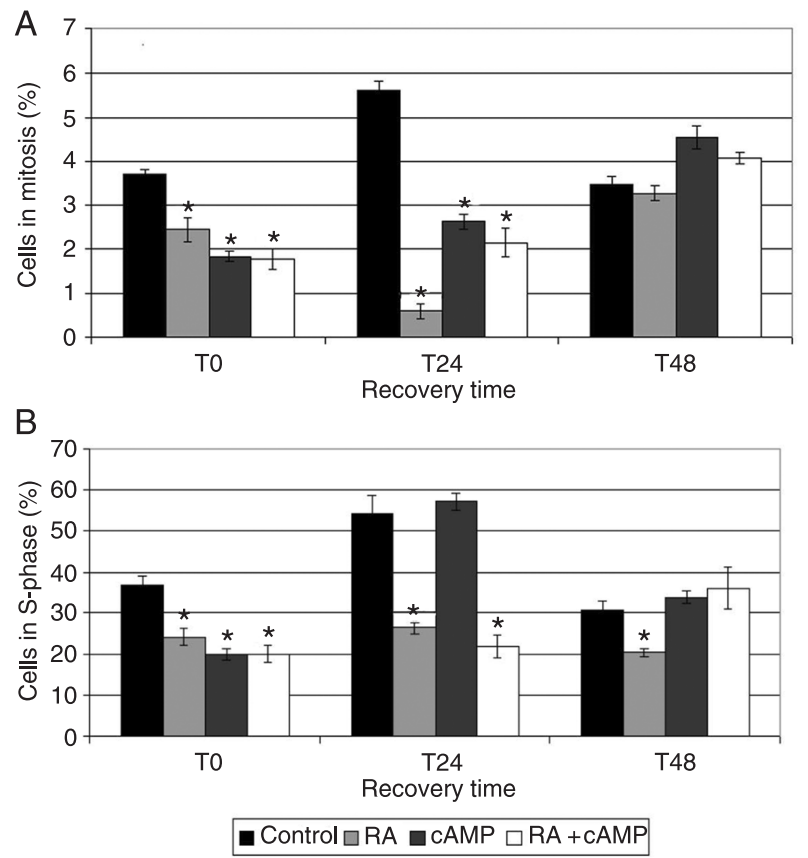

Figure 2. Cell proliferation behavior of hepatocellular carcinoma (HCC) cells as indicated by mitotic index $(A)$ and S-phase cell population $(B)$. Data are reported as $\%$ total cells. Mitotic indexes were significantly lower in cultures treated with retinoic acid (RA), cAMP and RA plus CAMP when compared to the control culture at T0 and T24. S-phase cell population frequencies were lower in all treated cultures compared to control at T0. At T24, cultures treated with RA alone and RA plus cAMP presented lower S-phase populations compared to control. At T48, only cultures treated with RA presented lower S-phase population than control. ${ }^{*} \mathrm{P}<0.05$, compared to control (Bonferroni test). 
The pattern of microtubule distribution changed according to treatment. This was clearly shown in the projections of Z-stack optical slices from the confocal LSM in a single plane image (Figure 4). The microtubules were seen more clearly in treated cells than in control cells. Both RA and cAMP changed the organization pattern of microtubules.

Cell adhesion to the substrate was improved by the treatments. We observed that both RA and cAMP increased the
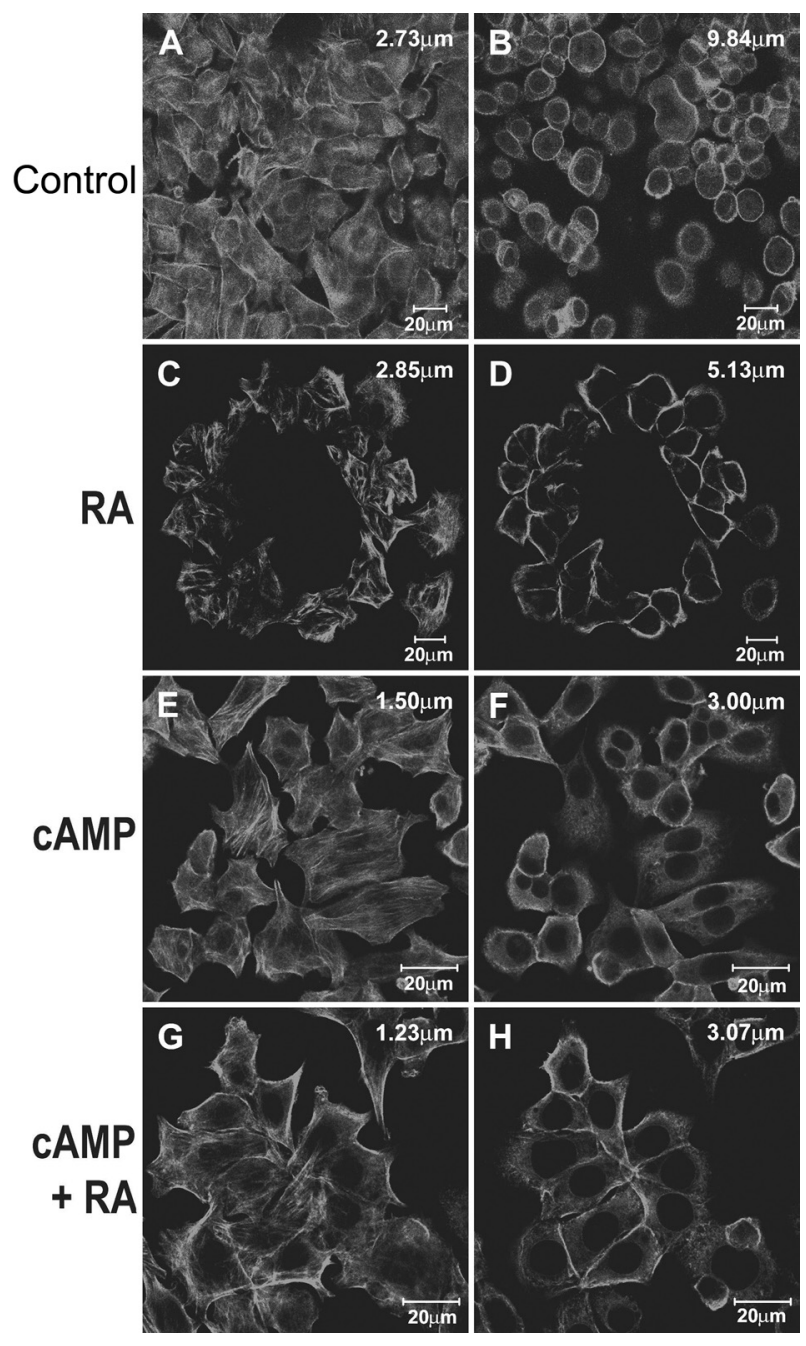

Figure 3. Laser scanning confocal microscopy images of HTC cells showing the pattern of actin filament distribution at T48. Left and right panels represent different optical sections on the Z-axis obtained during image capture: control cells $(A$ and $B)$, retinoic acid $(C$ and $D)$, CAMP ( $E$ and $F$ ), and retinoic acid plus CAMP ( $G$ and $H$ ). In the control culture shown in $B$, it is possible to observe the presence of rounded cells growing partially adhered to the substrate, which were not observed in treated cultures $(C-H)$. The treatments induced actin reorganization (actin stress fiber formation) and changes in cell shape $(C, E$ and $G)$. Note the presence of binucleated cells in cultures treated with cAMP $(F)$. adhesion capacity of treated cells to the laminin substrate at T0 and T24 (Figure 5).

\section{Connexin, E-cadherin and vinculin expression}

HTC cells preferentially express Cx43, compared to low levels or absence of Cx32 or Cx26 expression. This pattern of connexin expression did not change at T0, but changes in connexin expression profiles were observed at T24 and T48. At T24, we detected expression of Cx32
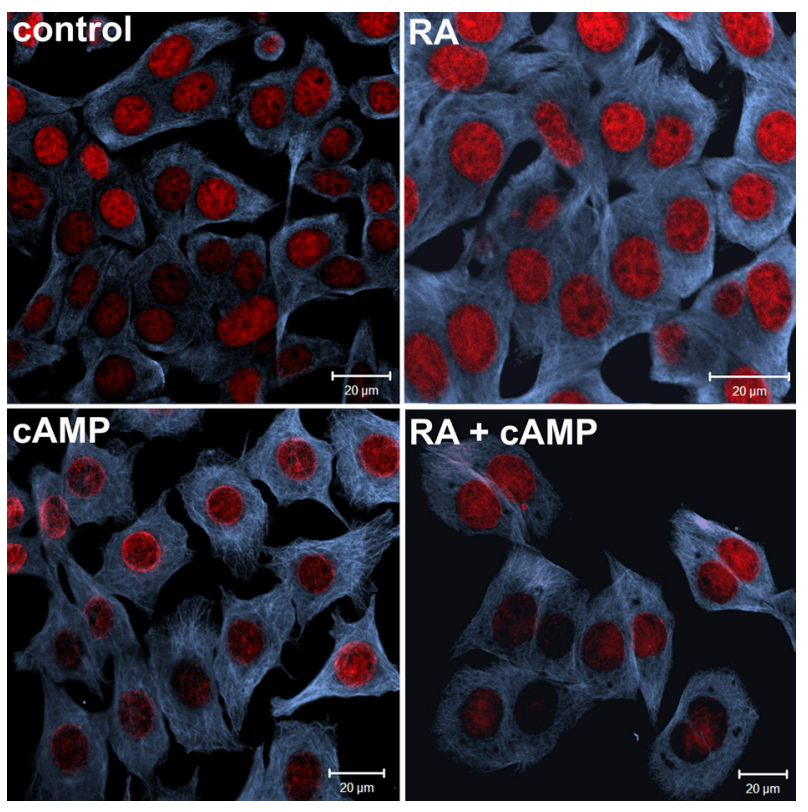

Figure 4. Images of HTC cells showing microtubule distribution at T48. The microtubule network is more evident in treated cells than in control cells, suggesting that retinoic acid (RA) and cAMP induced microtubule reorganization.

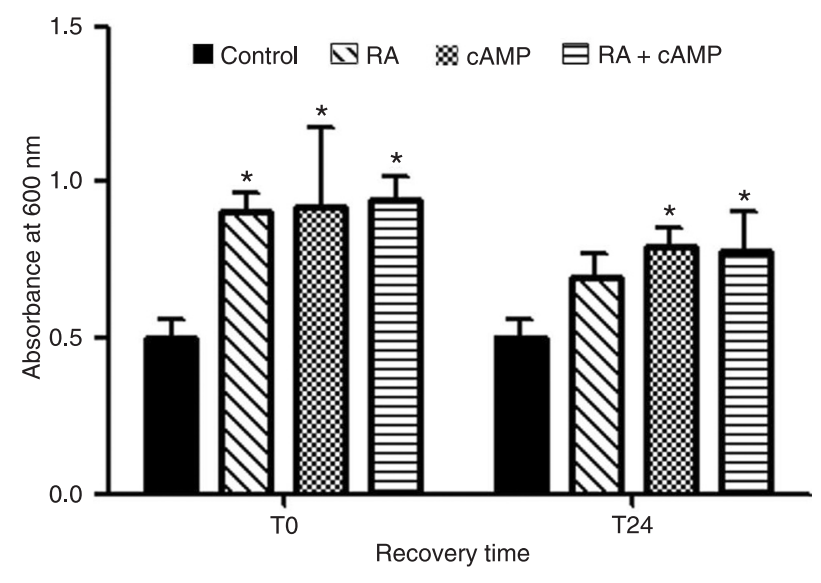

Figure 5. The adhesion assay using a laminin substrate showed increased cell-to-substrate interaction of treated cells. ${ }^{*} \mathrm{P}<0.05$ compared to control (Bonferroni test). 
in cultures treated with cAMP and RA plus cAMP. Also, Cx26 was expressed in all treated cells and its expression was highest in cells treated with cAMP at T24 (Figure 6). At T48, Cx32 expression was up-regulated in cells treated
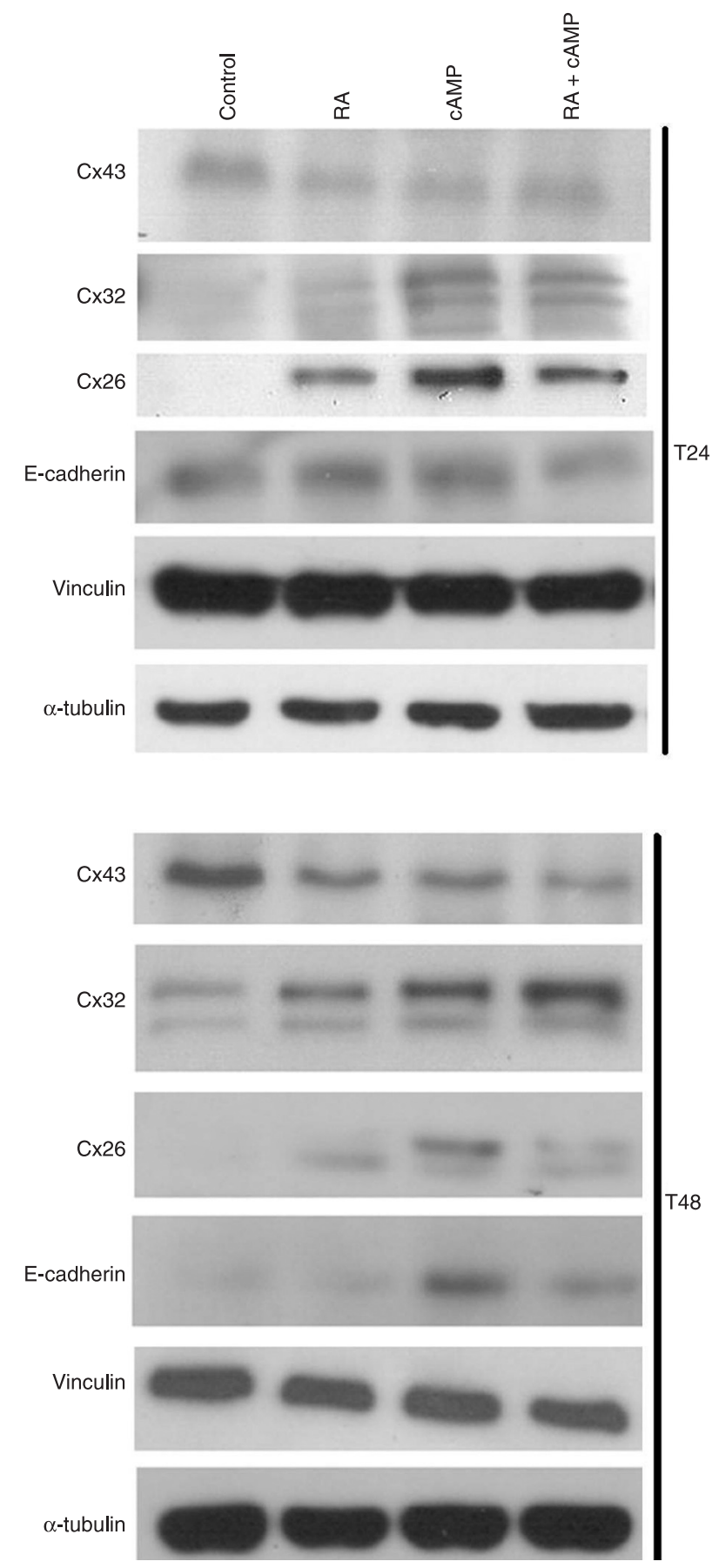

Figure 6. Immunoblot showing the expression pattern of connexins (Cx), E-cadherin and vinculin in treated and control cells. a-tubulin was used as the control for sample application. with cAMP, RA and RA plus cAMP. At the same time, Cx43 expression was down-regulated in all groups of treated cells (Figure 6). The treatments did not change the pattern of vinculin expression; however, at T24 we detected slightly increased E-cadherin expression in treated cultures, mainly in RA- and cAMP-treated cells. At T48, E-cadherin was preferentially expressed in CAMP-treated cells (Figure 6). Table 1 summarizes the modifications found in expression profiles in HTC cells treated with RA and cAMP.

\section{Telomerase activity}

Telomerase activity was another parameter associated with both cell proliferation capacity and cell differentiation status. The results obtained showed that both CAMP and RA can inhibit telomerase activity. cAMP presented strong inhibition capacity at T0 while RA was effective in regulating telomerase activity negatively at T48. Interestingly, telomerase activity was inhibited in cells treated with RA plus cAMP from T0 to T48. Figure 7 shows relative telomerase activity at the various recovery times.

\section{Expression of signaling proteins (ERK and GSK-3 $\beta$ )}

We investigated the expression profiles of total ERK, pERK and GSK-3 $\beta$ phosphorylated at Ser9 or Tyr216. All treated cells showed lower phosho-ERK expression levels compared to control cells. However, cells treated with cAMP showed decreased pERK expression compared with samples treated with RA and RA plus cAMP (Figure 8). We investigated the expression of both forms of GSK-3 $\beta$ (active and inactive), which corresponds to GSK-3 $\beta$ phosphorylated at Tyr216 and Ser9 residues, respectively. Ser9-GSK-3 $\beta$ expression levels were lower in control cells than treated cells. The cells treated with RA plus cAMP had the highest Ser9-GSK-3 $\beta$ expression levels (Figure 8 ). There were only slight changes in Tyr216-GSK-3 $\beta$ expression levels between control cells and cells treated with RA plus cAMP (Figure 8). Changes in the expression profiles of pERK and pGSK induced by the treatments are summarized in Table 1.

\section{Discussion}

In the present study, we investigated the effects of RA and/or cAMP on the proliferative behavior and phenotype of HTC cells. We also evaluated a possible synergistic effect between RA and cAMP. The treatment schedule was designed according to results obtained from BRL3A that showed high responsiveness to co-treatment. Therefore, HTC cells were treated for $48 \mathrm{~h}$ with RA or cAMP (in combination or not) followed by $24-$ and $48-\mathrm{h}$ recovery in fresh medium.

HTC cells treated with RA and cAMP exhibited changes both in cell morphology and in gene expression profiles that were consistent with a differentiated phenotype. It is important to note that these events were preferentially observed at the recovery times (T24 and T48), indicating that 
the effects of RA and CAMP on the proliferative behavior and phenotype of HTC cells continued after the treatment period. This aspect is very important to establish preclinical and clinical protocols that include RA and/or cAMP as chemotherapeutic agents.

During the recovery period we observed a decrease in Cx43 expression with a concomitant induction of Cx32 and Cx26 expression. This change in connexin expression profile occurred independently of the combined use of RA and cAMP; however, the highest Cx32 expression levels were observed at T48 in cultures treated with RA plus cAMP. In the liver, Cx43 is expressed by oval cells (hepatic stem-cells), endothelial cells and biliary cells, but not by hepatocytes. Cx32 and Cx26 are major connexin isoforms expressed by hepatocytes. Cx32 is expressed by hepatocytes of the hepatic acini while Cx26 is expressed by hepatocytes of the periportal spaces (17). Our results are very important because there are almost no data in the literature that demonstrate the modulation of connexin expression profile as a consequence of RA and/or cAMP treatment in hepatic tumor cells. We demonstrated that a mixture consisting of RA and cAMP was very effective in inducing Cx32 expression. Ara et al. (18) observed an increase of the $\mathrm{Cx} 32$ phosphorylated isoform in HepG2 cells after treatment with RA and they considered this event to be an important aspect of the reversal of the transformed phenotype of HepG2 cells.

In another independent study, Lee et al. (11) observed that HepG2 cells treated with cAMP for 3 days presented growth arrest as a consequence of both cyclin-dependent kinase (cdk) inhibitor up-regulation (p27Kip1, P21 Waf1/ Cip1) and cyclin A down-regulation, although they did not report morphological changes associated with a differentiated phenotype.

HepG2 was also used as a model by Alisi et al. (19), who detected growth inhibition of HepG2 cells after 7 days of treatment with RA. They demonstrated that RAinfluenced the activities of cyclin-cdk complexes involved in the regulation of G1/S transition and S-phase progression, mainly by modifying the binding of these complexes to p21 and p27 inhibitors. Thus, morphological analysis was not performed by Lee et al. (11) or by Alisi et al. (19), who worked independently to determine the effects of RA or cAMP on hepatic tumor cells. The present study shows for the first time the results obtained with the separate or concomitant use of $\mathrm{RA}$ and cAMP on the proliferative behavior and phenotype of hepatocarcinoma cells.

Telomerase activity is a hallmark of the immortal phenotype and its suppression has been considered an important parameter to detect the efficacy of RA in inducing cell differentiation and reversal of transformed phenotype in leukemia cells as well as in breast cancer and neuroblastoma cells (20-22). We determined that cAMP exerts a strong negative effect on telomerase activity, which was observed at T0. RA also inhibited telomerase activity; however, this effect
Table 1. Modifications of the expression profiles of HTC cells treated with retinoic acid (RA) and cAMP.

\begin{tabular}{lccc}
\hline & RA & cAMP & RA + cAMP \\
\hline Cx43 & $\downarrow$ & $\downarrow$ & $\downarrow$ \\
Cx32 & $\uparrow$ & $\uparrow$ & $\uparrow$ \\
Cx26 & $\uparrow$ & $\uparrow$ & $\uparrow$ \\
E-cadherin & Slightly $\uparrow$ & $\uparrow$ & $\uparrow$ \\
Vinculin & No alteration & No alteration & No alteration \\
pERK & $\downarrow$ & $\downarrow$ & $\downarrow$ \\
Ser9-GSK-3 & $\uparrow$ & $\uparrow$ & $\uparrow$ \\
Tyr216-GSK-3 $\beta$ & Slightly $\downarrow$ & Slightly $\downarrow$ & Slightly $\downarrow$ \\
\hline
\end{tabular}

The quantitative analysis was performed using ImageJ, a public domain program. $\mathrm{Cx}=$ connexin; $\mathrm{pERK}=$ phosphorylated extracellular signal-regulated kinase; GSK-3 $\beta=$ glycogen synthase kinase 3 beta.

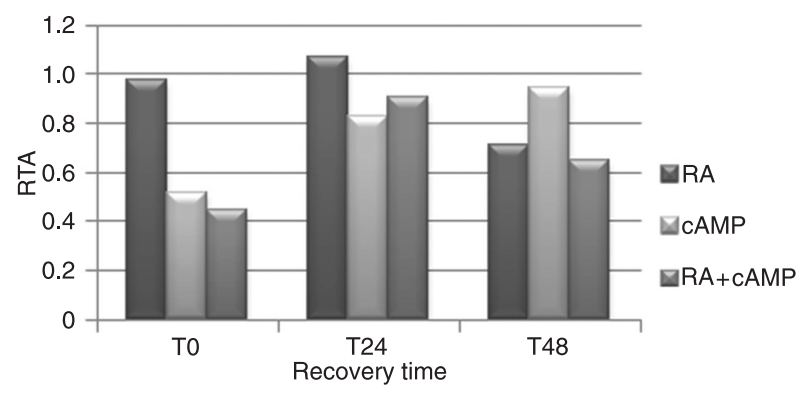

Figure 7. Relative telomerase activity (RTA) was determined using the TeloTAGGG Telomerase PCR ELISAPLUS kit and was calculated according to the following formula: RTA $=[$ (Abs sample - Abs RNAase-treated sample) / Abs internal standard of the sample] / [(Abs control template - Abs lysis buffer) / Abs internal standard of the control template $] \times 100$, where Abs = absorbance. $\mathrm{RA}=$ retinoic acid.
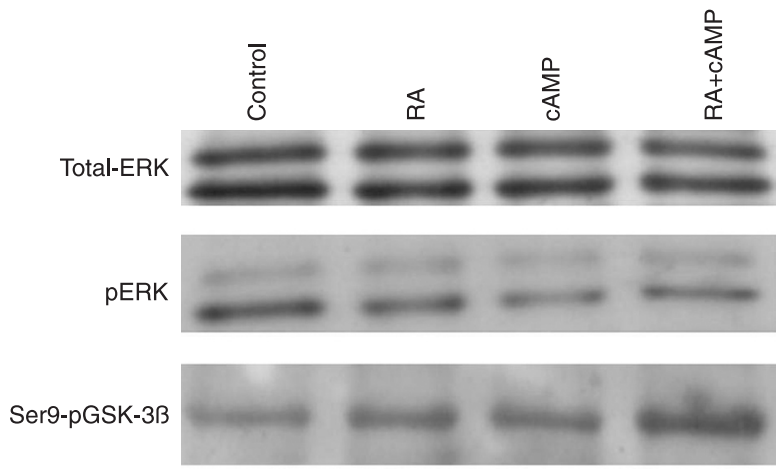

Tyr216-pGSK-3ß

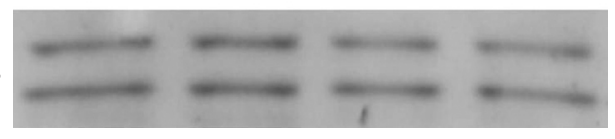

Figure 8. Immunoblot showing the expression patterns of signaling extracellular signal-regulated kinase (ERK) and glycogen synthase kinase 3 beta (GSK-3 $\beta$ ) protein at T24. pERK = phosphorylated $E R K ; R A=$ retinoic acid. Total- ERK was the sample loading control. 
was only seen at T48. Thus, cells treated with the mixture (RA plus CAMP) presented inhibition of telomerase activity from T0 to T48. The concomitant use of RA and cAMP can be useful to improve the effect of treatment, mainly with low RA concentrations. In this study, for example, RA was used at $0.1 \mu \mathrm{M}$.

Treatment with RA and/or cAMP promoted microfilament reorganization in HTC cells. This event seems to be critical to cell shape modification because the treated cells became flattened, exhibited polyhedric morphology and presented higher adhesion capacity, both cell-to-cell and cell-to-substratum. It is possible that the protein complexes involved in intercellular contacts and cell polarity were stabilized by cytoskeleton reorganization. We saw important up-regulation of E-cadherin expression in cultures treated with CAMP, even though we did not observe changes in vinculin expression in these cultures. Similar data were obtained by Masuda et al. (23), who observed E-cadherin up-regulation in hepatocellular carcinoma cells (HCC-T and HCC-M) treated with sodium butyrate.

Assays for cell adhesion to a laminin substrate confirmed the effect of treatment. After treatment with RA and/or cAMP, the cells presented higher adhesion capacity than untreated cells. Good adhesive ability may be a relevant and appropriate characteristic of well-differentiated cells. Our data agree with Massimi and Divirgiliis (24), who demonstrated increased adhesiveness of HepG2 cells after treatment with RA for 12 days. A similar experimental approach has not been previously used with cAMP. Considering that the RAF/ MEK/ERK pathway is constitutively activated in HCC (2527), we investigated whether the drugs used in this study had some effect on ERK phosphorylation. We observed lower pERK expression levels in treated cells compared to control cells. This result suggests that the antiproliferative effects of RA and cAMP on HTC cells may be due, at least in part, to their capacity to negatively regulate ERK phosphorylation. Similar results were observed by Kanamori et al. (28), who showed cell proliferation inhibition by acriflavine, a synthetic retinoid, with concomitant ERK phosphorylation inhibition in human $\mathrm{HCC}$ cells ( $\mathrm{HuH} 7)$.

In the present study, treatment with cAMP was more

\section{References}

1. Ferlay J, Shin HR, Bray F, Forman D, Mathers C, Parkin DM. Estimates of worldwide burden of cancer in 2008: GLOBOCAN 2008. Int J Cancer 2010; 127: 2893-2917.

2. Wong $\mathrm{CM}, \mathrm{Ng} \mathrm{IO}$. Molecular pathogenesis of hepatocellular carcinoma. Liver Int 2008; 28: 160-174.

3. Noy N. Between death and survival: retinoic acid in regulation of apoptosis. Annu Rev Nutr 2010; 30: 201-217.

4. Bushue N, Wan YJ. Retinoid pathway and cancer therapeutics. Adv Drug Deliv Rev 2010; 62: 1285-1298.

5. Wang ZY, Chen Z. Acute promyelocytic leukemia: from highly fatal to highly curable. Blood 2008; 111: 2505-2515. effective in inhibiting ERK phosphorylation than RA treatment or co-treatment. Thus, we extended our study and also investigated the effect of these drugs on GSK-3 $\beta$, whose activity is modulated by several signaling pathways including the Wnt, PI-3K/AKT and Ras/MAPK pathways (29). GSK$3 \beta$ activity is regulated by site-specific phosphorylation of Tyr216/Ser9 residues (30). The active and inactive forms are characterized by a phosphorylation pattern at the Tyr216 and Ser9 residues, respectively. GSK-3 $\beta$ drives oncogenic progression either by its inhibition or its activation, depending on the tumor (31). In gastrointestinal cancers (pancreatic, colorectal and hepatic), lower pSer9-GSK-3 $\beta$ expression is commonly observed compared to the active form (32). In fact, we observed low pSer9-GSK-3 $\beta$ expression in control cells while in treated cells pSer9-GSK- $3 \beta$ expression levels were up-regulated. Interestingly, cells treated with RA plus cAMP exhibited higher pSer9-GSK-3 $\beta$ expression than cells treated with only RA or CAMP.

The interaction of drugs with the cellular environment is extremely complex and requires extensive investigation. The exploration of the recovery times permitted us to evaluate in more detail a possible interaction between RA and cAMP. For example, if we evaluated independently each time we could conclude that there is no synergistic effect between RA and cAMP. However, when we analyzed the kinetics of the events along the recovery time we noted that co-treatment contributed to extending the period during which cell growth was inhibited and increased the degree of HTC cell differentiation.

The present study demonstrated that RA and cAMP can inhibit cell proliferation and are also effective in inducing differentiation of HTC cells. However, when they are used in combination the signals regarding cell proliferation inhibition are temporally amplified and the degree of cell differentiation is increased.

\section{Acknowledgments}

The authors thank Roberto Cabado Modia for assistance with confocal imaging and the preparation of the figures. Research supported by FAPESP, CNPq, and CAPES/PNPD.

6. Wang A, Zeng $R$, Huang $H$. Retinoic acid and sodium butyrate as cell cycle regulators in the treatment of oral squamous carcinoma cells. Oncol Res 2008; 17: 175-182.

7. Ventura-Holman T, Mamoon A, Subauste JS. Modulation of expression of RA-regulated genes by the oncoprotein v-erbA. Gene 2008; 425: 23-27.

8. Lu J, Zhang F, Zhao D, Hong L, Min J, Zhang L, et al. ATRAinhibited proliferation in glioma cells is associated with subcellular redistribution of beta-catenin via up-regulation of Axin. J Neurooncol 2008; 87: 271-277.

9. Jung HY, Park SH, Yoo YD, Kim JS, Kim YH. CDK2/4 
regulate retinoic acid-induced G1 arrest in hepatocellular carcinoma cells. Hepatol Res 2005; 31: 143-152.

10. Quenech'Du N, Ruchaud S, Khelef N, Guiso N, Lanotte $M$. A sustained increase in the endogenous level of cAMP reduces the retinoid concentration required for $\mathrm{APL}$ cell maturation to near physiological levels. Leukemia 1998; 12: 1829-1833.

11. Lee J, Choi YH, Nguyen P, Kim JS, Lee SJ, Trepel JB. Cyclic AMP induces inhibition of cyclin A expression and growth arrest in human hepatoma cells. Biochim Biophys Acta 1999; 1449: 261-268.

12. Liu L, Xie Y, Lou L. Cyclic AMP inhibition of proliferation of hepatocellular carcinoma cells is mediated by Akt. Cancer Biol Ther 2005; 4: 1240-1247.

13. Kovach SJ, Price JA, Shaw CM, Theodorakis NG, McKillop $\mathrm{IH}$. Role of cyclic-AMP responsive element binding (CREB) proteins in cell proliferation in a rat model of hepatocellular carcinoma. J Cell Physiol 2006; 206: 411-419.

14. Thompson EB, Tomkins GM, Curran JF. Induction of tyrosine a-ketoglutarate transaminase by steroid hormones in a newly established tissue culture cell line. Biochemistry 1966; 56: 296-303

15. Prado MP, Torres YR, Berlinck RGS, Desidera C, Sanchez MA, Craveiro MV, et al. Effects of marine organisms extracts on microtubule integrity and cell cycle progression in cultured cells. J Exp Mar Biol Ecol 2004; 313: 125-137.

16. Machado-Santelli GM, Mori L, Pereira CA. Prediction of relapse in patients with breast cancer by DNA cytometry. Anal Cell Pathol 1994; 7: 321-334.

17. Neveu MJ, Sattler CA, Sattler GL, Hully JR, Hertzberg EL, Paul DL, et al. Differences in the expression of connexin genes in rat hepatomas in vivo and in vitro. Mol Carcinog 1994; 11: 145-154.

18. Ara C, Massimi M, Devirgiliis CL. Retinoic acid modulates gap junctional intercellular communication in hepatocytes and hepatoma cells. Cell Mol Life Sci 2002; 59: 17581765.

19. Alisi A, Leoni S, Piacentani A, Conti DL. Retinoic acid modulates the cell-cycle in fetal rat hepatocytes and HepG2 cells by regulating cyclin-cdk activities. Liver Int 2003; 23: 179186.

20. Yamada O, Ozaki K, Nakatake M, Akiyama M, Kawauchi $\mathrm{K}$, Matsuoka R. Multistep regulation of telomerase during differentiation of HL60 cells. J Leukoc Biol 2008; 83: 12401248.

21. Phipps SM, Love WK, White T, Andrews LG, Tollefsbol TO.
Retinoid-induced histone deacetylation inhibits telomerase activity in estrogen receptor-negative breast cancer cells. Anticancer Res 2009; 29: 4959-4964.

22. Das A, Banik NL, Ray SK. Retinoids induce differentiation and downregulate telomerase activity and N-Myc to increase sensitivity to flavonoids for apoptosis in human malignant neuroblastoma SH-SY5Y cells. Int J Oncol 2009; 34: 757-765.

23. Masuda T, Saito H, Kaneko F, Atsukawa K, Morita M, Inagaki $\mathrm{H}$, et al. Up-regulation of E-cadherin and I-catenin in human hepatocellular carcinoma cell lines by sodium butyrate and interferon-alpha. In Vitro Cell Dev Biol Anim 2000; 36: 387394.

24. Massimi M, Devirgiliis LC. Adhesion to the extracellular matrix is positively regulated by retinoic acid in HepG2 cells. Liver Int 2007; 27: 128-136.

25. McKillop IH, Schmidt CM, Cahill PA, Sitzmann JV. Altered expression of mitogen-activated protein kinases in a rat model of experimental hepatocellular carcinoma. Hepatology 1997; 26: 1484-1491.

26. Ito $Y$, Sasaki $Y$, Horimoto M, Wada S, Tanaka $Y$, Kasahara A, et al. Activation of mitogen-activated protein kinases/extracellular signal-regulated kinases in human hepatocellular carcinoma. Hepatology 1998; 27: 951-958.

27. Feng DY, Zheng $\mathrm{H}$, Tan $\mathrm{Y}$, Cheng RX. Effect of phosphorylation of MAPK and Stat3 and expression of c-fos and c-jun proteins on hepatocarcinogenesis and their clinical significance. World J Gastroenterol 2001; 7: 33-36.

28. Kanamori T, Shimizu M, Okuno M, Matsushima-Nishiwaki $\mathrm{R}$, Tsurumi H, Kojima S, et al. Synergistic growth inhibition by acyclic retinoid and vitamin K2 in human hepatocellular carcinoma cells. Cancer Sci 2007; 98: 431-437.

29. Cohen P, Frame S. The renaissance of GSK3. Nat Rev Mol Cell Biol 2001; 2: 769-776.

30. Doble BW, Woodgett JR. GSK-3: tricks of the trade for a multi-tasking kinase. J Cell Sci 2003; 116: 1175-1186.

31. Kang T, Wei Y, Honaker Y, Yamaguchi H, Appella E, Hung MC, et al. GSK-3 beta targets Cdc25A for ubiquitin-mediated proteolysis, and GSK-3 beta inactivation correlates with Cdc25A overproduction in human cancers. Cancer Cell 2008; 13: 36-47.

32. Mai W, Kawakami K, Shakoori A, Kyo S, Miyashita K, Yokoi $\mathrm{K}$, et al. Deregulated GSK3 $\{$ beta\} sustains gastrointestinal cancer cells survival by modulating human telomerase reverse transcriptase and telomerase. Clin Cancer Res 2009; 15: 6810-6819. 